\title{
Palm oil classification using deep learning
}

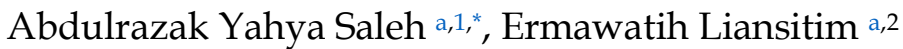 \\ a Faculty of Cognitive Science and Human Development, Universiti Malaysia Sarawak, \\ 94300 Kota Samarahan, Sarawak, Malaysia \\ 1 ysahabdulrazak@unimas.my \\ * Corresponding Author
}

\section{ARTICLE INFO}

Article history

Received March 8, 2019

Revised June 11, 2019

Accepted January 10, 2020

Keywords

Classification

Deep Convolutional

Neural Network

Palm Oil Ripeness

\begin{abstract}
Deep Convolutional Neural Networks (CNNs) have been established as a dominant class of models for image classification problems. This study aims to apply and analyses the accuracy of deep learning for classifying ripes on palm oil fruit. The CNN used to classify 628 images into 2 different classes. Furthermore, the experiment of CNN with 5 epochs gives promising classification results with an accuracy of $98 \%$, which is better than previous methods. To sum up, this study was successfully solving an image classification by detected and differentiated the ripeness of oil palm fruit.
\end{abstract}

This is an open access article under the CC-BY-SA license.

\section{Introduction}

The palm oil plant (Elaeis Guineensis) is one of the typical species found in Malaysia [1]. Basically, this species is the common African palm oil and mostly used as a cooking oil [2]-[5]. The Malaysian palm oil industry is tightly regulated. The main problem for palm oil exporters and producers before processing is accuracy in assessing the freshness of oil palm according to its maturity level [6]. The maturity of palm fruit is quality and is the selling power of the palm oil produced. In Malaysia, palm oil is the primary agricultural product industry [7]. In addition, palm oil production and exports in Malaysia account for $41 \%$ of world production and $47 \%$ of world exports [8]. Therefore, as an export commodity, the production of good quality oil palm is vital because most of the import countries are quality conscious customer [9]. Thus, in getting the quality and quantity of oil products, it is essential in identifying the maturity of FFB, thereby increasing profits for the industry.

Currently, fruit bunches grading is manually applied in palm mills, where the grader will follow the standard inspection given by authorities based on oil palm maturity if ripe and unripe [10]. However, the human examination is very subjective depending on the different human grading classifies differently, and the expert's expertise may fail to articulate the grading criteria correctly [7]. Generally, fruit bunches will be grade dependent on the color of the fruit. As a human, we see things differently alike human grader also sees fruit bunches from different colors. This practice is often leading to disputes between graders and sellers. Therefore, it is important to find a solution in determining the maturity of the oil palm fruit.

Research on automatic grading systems is becoming a future trend because it has a high potential for new approaches [11]. Many studies of oil palm fruit have been published. The 
research applied several techniques, and most of them used machine vision. Currently, the image classification approach uses machine learning methods.

To improve their performance, we can collect larger datasets, learn more powerful models, and use better techniques for preventing overfitting. Therefore, this study aims to develop a new technique that can assist the classification for palm oil fruit and act like a human who is able to recognize the color and make a decision based on the selected category. The technique that was developed is using CNN as it's a good classifier in image classification and recognition tasks, as well as for the problem of fruit recognition [8].

\section{Method}

In addition, CNN can learn spontaneously from the input and extracts global features and contextual details, which reduces error slightly in image recognition.

\subsection{Methodology Flow}

This study uses a sample of two categories, namely ripe and immature. This research identifies the color features of oil palm fruit so that the analysis of the texture and shape of oil palm bunches is not needed. The flow chart in identifying the maturity of oil palm fruit is shown as in Fig. 1, which provides detailed identification steps.

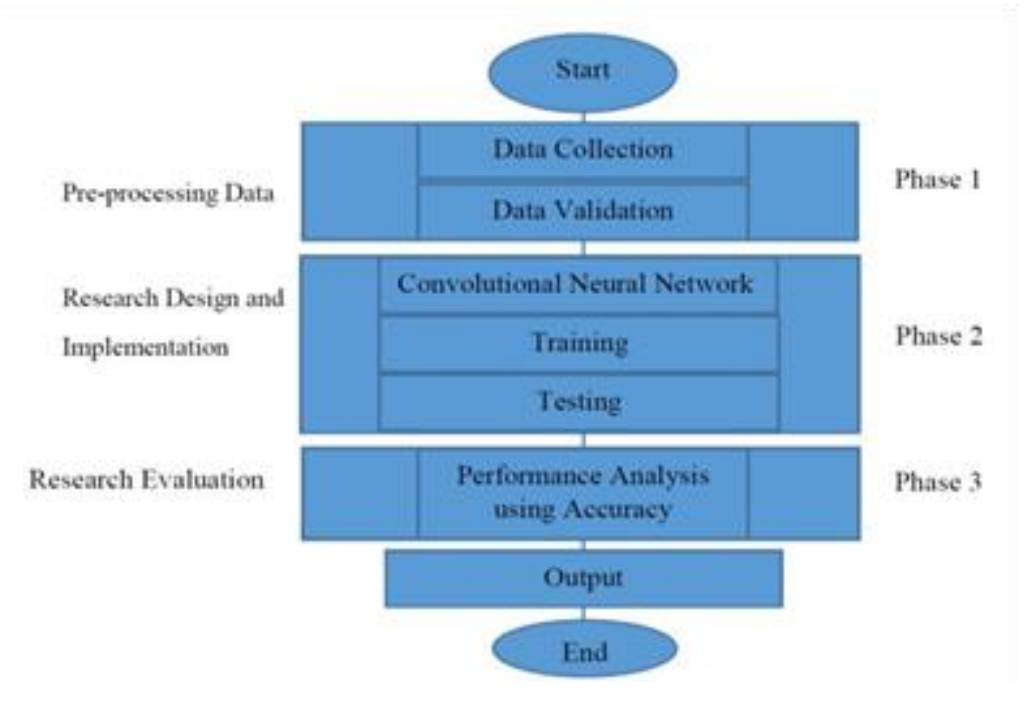

Fig. 1. General Framework

\subsection{Data Collection and Preparation}

The datasets for this study were collected from the Tomanggong Palm Oil Mill, Lahad Datu Sabah. In this study, the standard dataset is used as a sample to test the effectiveness of the Convolutional Neural Network as a classifier. The sample of the original data for each bunch is presented in Fig. 2 and Fig. 3. 


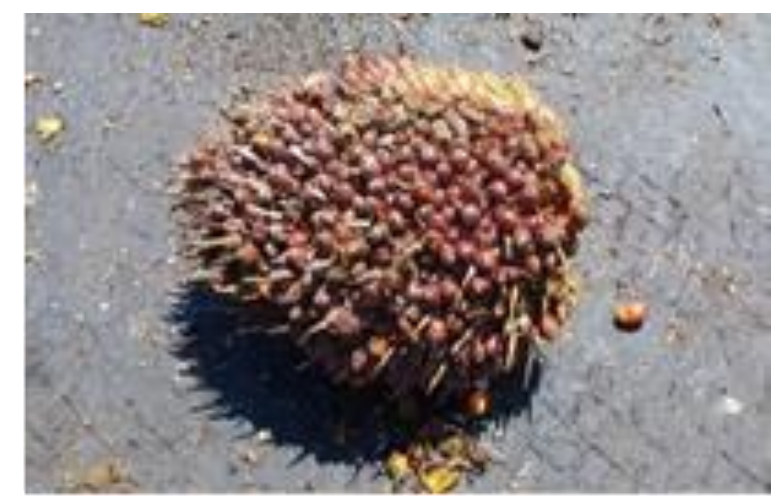

Fig. 2. Oil Palm Fruit Bunch-Ripe

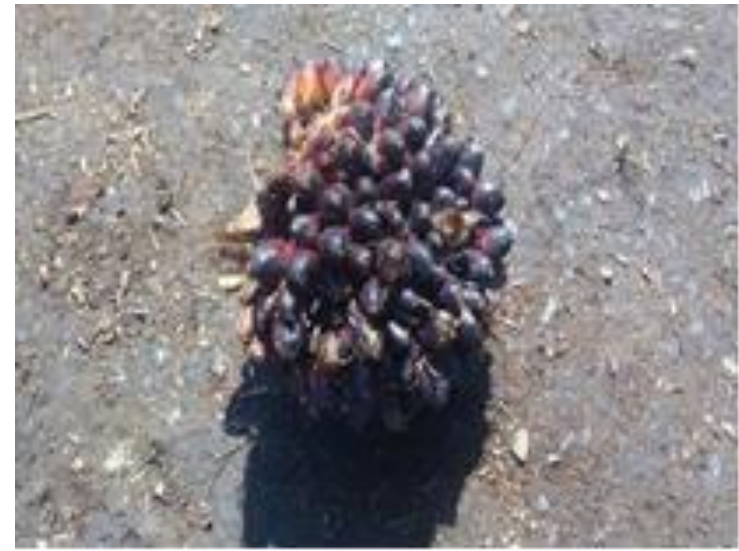

Fig. 3. Oil Palm Fruit Bunch-Unripe

In this study, the holdout method is used to validate the datasets. The datasets of this study were assigned into two points of the set, which are $\mathrm{d} 0$ and $\mathrm{d} 1$. This method is a training set, and testing data is respected. Each set size is arbitrary, with the test set used smaller than the training set. The training process is on $\mathrm{d} 0$, and testing is on $\mathrm{d} 1$. The holdout is also used to determine the algorithm's predictive prowess and provides a final estimate of model performance after it has been trained and validated [12].

\subsection{Research Design and Implementation}

CNN is used as a classifier of the ripeness of oil palm fruit. The essential instinct behind these frameworks is that a processing architecture based on a huge number of layered and massively interconnected simple units may be fit than sophisticated algorithms to handle complex issues. The fundamental processing unit, the neuron, is exceptionally basic. It calculates the output activation by looking at the weighted entirety of its contribution with a threshold and applying a suitable nonlinearity, below is (1).

$$
\mathrm{O}_{j}=\varnothing\left(\sum_{i} w_{i j} x_{i}-\theta_{j}\right)
$$

CNN training requires input images and related labels and automatically extracts image features. The purpose of the training algorithm is to train the network in input and layer by layer so that errors are minimized between the network output and desired output or to improve output performance. Neural network architects are made with an additional layer called the loss layer. This layer is a critique of neural networks if it recognizes the source of information effectively, and if not, how far guessed. It can reinforce the right ideas when trained. The CNN performance validation process uses validation data without a training process when the validation performance does not improve. Fig. 4 is an example of a training set. 


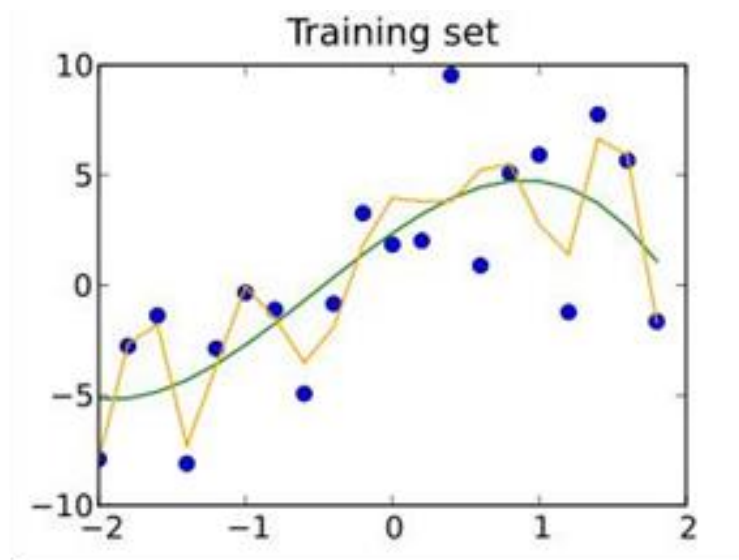

Fig. 4. Training Set

In the training process, the mean squad error (MSE) of the fit on the orange line is 4, while the MSE for fit on the green line is 9. The training data that is very suitable is the orange curve because the MSE value increases by almost a factor of four if compared between testing and training. Green curves are not suitable for training data, because the MSE increases by less than a factor of 2 .

The data set testing technique is called the data augmentation technique. The training process is shown in Fig. 5, which is a validation test of the model being built. Data points in the training set are ignored from the test (validation) [13]. For each iteration, the data is separated into validation sets and test sets. The calculation process in the training set determines various coefficients. Data can be tested to predict based on broader data sets and provide information about errors. MSE for fit on the orange line is 15, and MSE for fit on the green line is 13.

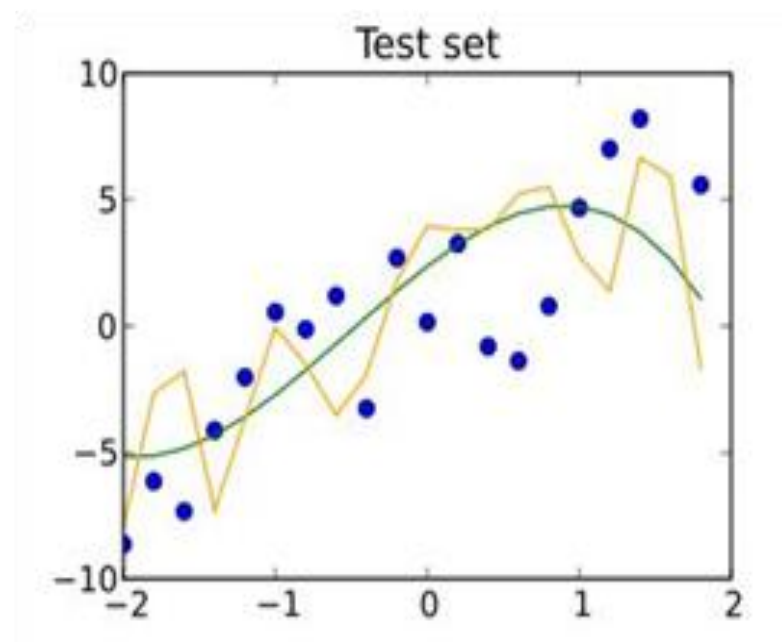

Fig. 5. A test set result activity while built a model of datasets using keras

\section{Results and Discussion}

\subsection{Research Evaluation}

This accuracy is used to evaluate the effectiveness of the algorithm to classify the images of ripe and unripe palm oil fruits. This accuracy will be proved the effectiveness of CNN in categorizing palm oil ripeness. The accuracy was calculated by (2). 


$$
\text { Accuracy }=\frac{\mathrm{TP}}{\mathrm{TP}+\mathrm{TN}} \times 100 \%
$$

Where TP is true positive, and TN is true negatives. TP and TN are using for correct classification. A false positive (FP) is when the outcome of the algorithm is incorrectly predicted, whereas False positive (FP) is used when the predicted results do not match.

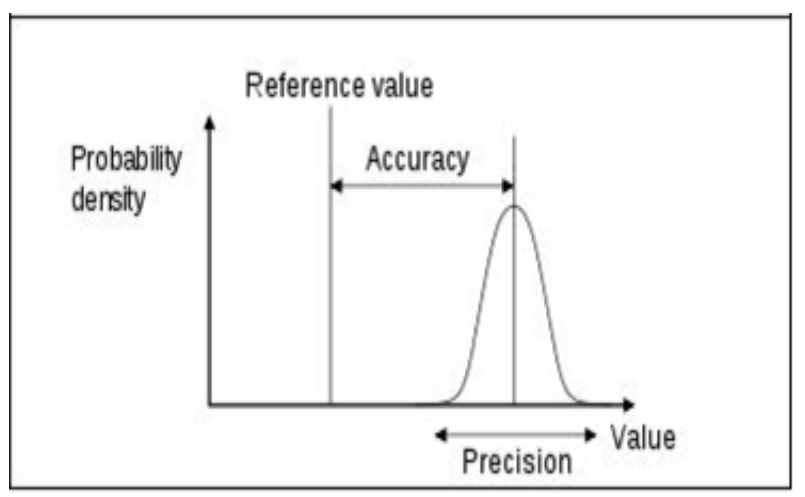

Fig. 6.Training A test set result activity while built a model of datasets using keras ng Set

Based on Fig. 6, the closeness of the measurement results to the true value, precision, repeatability, or reproducibility of the measurement is a factor of accuracy. Besides, to determine classification accuracy depends on the size of the convolutional kernel and maxpooling kernel, the number of kernels in each convolutional layer, and hidden units in the fully connected layer [2], [14].

\subsection{Results}

The keras deep learning library that has been used to train the images stored in the folder. The result shows when the image is analyses, which the result equal to 1 . From the coding, 1 belongs to unripe. In this study, 5 epochs were used to determine the best accuracy for training and testing datasets. Each of epochs stated the loss accuracy and validation accuracy. By observing the training accuracy and loss, after 5 epochs, the final accuracy for training accuracy is $96 \%$, and the training loss is quite low, which is 0.5295 . The validation accuracy is $97 \%$, with validation loss 0.0476 . The result shows if the result [0][0] $=1$, then the prediction is unripe, else would be ripe. In this study, the result is 1 , which is the images belonged to unripe. Table 1 shown below is the training result at each of single epoch. The end of the epochs showed the result of ripeness, which 1 belongs to unripe. This study was successfully classified images of palm oil by detected and differentiated the ripeness of oil palm fruit.

Table 1. Findings of training and testing for each epoch

\begin{tabular}{ccccc}
\hline Epoch & Loss & Accuracy & Validation Loss & Validation Accuracy \\
\hline $1 / 5$ & 0.5295 & $73 \%$ & 0.3864 & $81 \%$ \\
\hline $2 / 5$ & 0.3865 & $82 \%$ & 0.3184 & $86 \%$ \\
\hline $3 / 5$ & 0.2634 & $89 \%$ & 0.1843 & $94 \%$ \\
\hline $4 / 5$ & 0.1626 & $93 \%$ & 0.1055 & $96 \%$ \\
\hline $5 / 5$ & 0.0971 & $96 \%$ & 0.0476 & $97 \%$ \\
\hline Result & $1=$ unripe & & \\
\hline
\end{tabular}




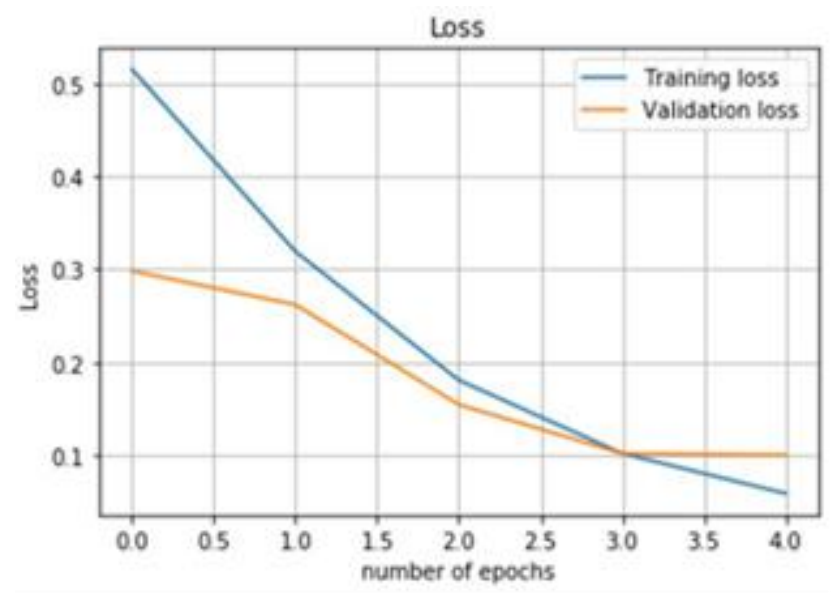

Fig. 7. The error of datasets during training and test the models using Python

Fig. 7 is a result of training loss and validation loss of CNN performance. From the figure, we can see that the model performance is decreased from epoch to the next epoch, which is the error for both datasets are decreased at every level of the epoch. Parallel validation dataset plots according parts consistently; it was a sign of training stopping at the earlier epoch.

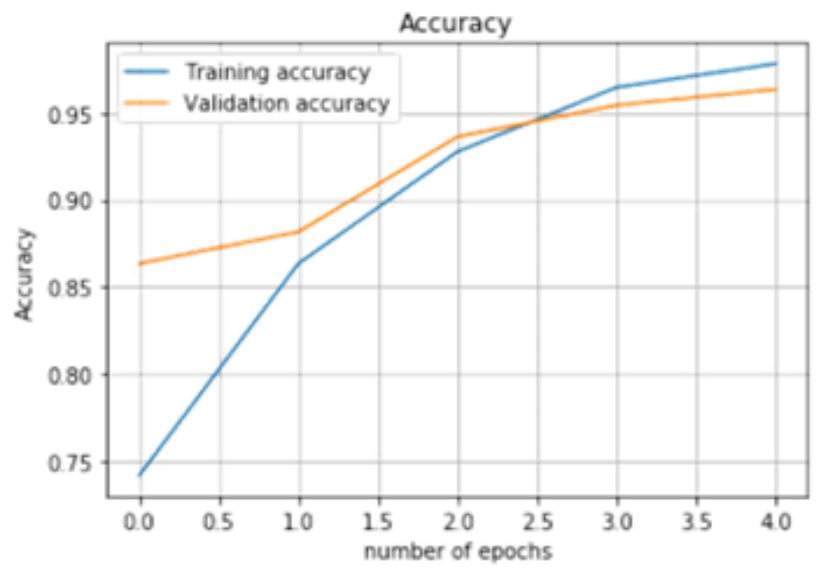

Fig. 8. The training and validation accuracy of palm oil fruit using Keras

Fig. 8 represented the result of the accuracy of training and validation or test datasets. The accuracy of the training process and validation has not been much learned from the training dataset. Comparable skills show this in the two datasets. Model accuracy can be trained more to get increased accuracy in both data sets.

\subsection{Discussion}

Convolutional neural networks are created for the learning process with large amounts of data [15]. The palm oil FFB maturity classification algorithm has been implemented. This article proposes a machine learning framework for assessing oil palm fruit bunches using the Convolutional Neural Network algorithm. Detection of maturity is based on a lack of productivity, efficiency problems, and is still assessed by human visualization [6]. This project aims to determine and differentiate the color characteristics of oil palm fruit bunches.

This study was successfully solving an image classification by detected and differentiated the ripeness of oil palm fruit. The three objectives were achieved, which is the first objective is to pre-process the data set of palm oil fruit. By using this algorithm, the datasets have been preprocessed to be suitable for the classification of palm oil fruit. 
Secondly, to apply deep learning in classifying palm oil fruit, The CNN that applied in this study can learn the datasets which show where the class input image belongs to either it ripe or unripe.

The third objective is to analyses the effectiveness of applying deep learning in classifying palm oil fruit. It was achieved where CNN can learn better from the input, and it can predict the ripeness of palm oil fruit either it is ripe or unripe effectively with an accuracy of $98 \%$. The way those objectives achieved by training a CNN on few images of ripe and unripe palm oil and make CNN learn to predict which class the image belongs to. The computer software used is Python 3.2.5, which is used to analyze image data. This research provides benefits to industry, engineers, harvesters, appraisers, mill operators, plantation managers, small farmers, and the research community related to oil palm.

The result of this study, if compare with the other research that used CNN on image classification, such as Cheng et al. [16] achieved 96\% accuracy of correct classification samples. The researcher mentioned in her study that 10 epochs are enough for successful training of the model. This study also compares CNN algorithm with other methods that have been conducted before to classifying palm oil ripeness [6] has been conducted a study on oil palm fruit grading using a hyperspectral device and machine learning algorithm which the result of the classification approach of the study had an accuracy of more than $95 \%$ for all three types of the oil palm fruit. While in this study approach which using the CNN algorithm achieved 96\% accuracy than the ANN model. The researchers conclude that the CNN algorithm is more effective in classifying images than the ANN algorithm.

\section{Conclusion}

This study has been proven, which has high accuracy in image classification using CNN algorithm. This technique also used to train and test the images that have been stored in folder. However, given the limitations of the dataset and time, future research can add datasets to improve results. Furthermore, using only test data with a single image, the discovery of similar results at a more significant amount can be strengthened. The good overall result of this study, it can predict the image of palm oil. To improve the performance of this method, future work must get more data because deep learning algorithms often perform better with more data and usually, the quality of models is generally constrained by the quality of the training data [17], [18]. The future assessment system can be connected to the internet so that users can test the maturity of the fruit online (future research).

\section{Acknowledgment}

This research is supported and funded by RIMC of University Malaysia Sarawak (UNIMAS), under the special Grant Scheme (F04/SpGS/1547/2017).

\section{References}

[1] E. Onoja, S. Chandren, F. I. Abdul Razak, N. A. Mahat, and R. A. Wahab, "Oil Palm (Elaeis guineensis) Biomass in Malaysia: The Present and Future Prospects," Waste and Biomass Valorization, vol. 10, no. 8, pp. 2099-2117, Aug. 2019, doi: 10.1007/s12649-018-0258-1.

[2] A. Manandhar, L. Hoegner, and U. Stilla, "Palm tree detection using circular autocorrelation of polar shape matrix," ISPRS Ann. Photogramm. Remote Sens. Spat. Inf. Sci., vol. III-3, pp. 465-472, Jun. 2016, doi: 10.5194/isprs-annals-III-3-465-2016.

[3] E. Khankhaje et al., "On blended cement and geopolymer concretes containing palm oil fuel ash," Mater. Des., vol. 89, pp. 385-398, Jan. 2016, doi: 10.1016/j.matdes.2015.09.140.

[4] A. Mancini et al., "Biological and nutritional properties of palm oil and palmitic acid: effects on health," Molecules, vol. 20, no. 9, pp. 17339-17361, Sep. 2015, doi: 10.3390/molecules200917339.

[5] S. Yue, J. F. Brodie, E. F. Zipkin, and H. Bernard, "Oil palm plantations fail to support mammal 
diversity," Ecol. Appl., vol. 25, no. 8, pp. 2285-2292, Dec. 2015, doi: 10.1890/14-1928.1.

[6] O. M. Bensaeed, A. M. Shariff, A. B. Mahmud, H. Shafri, and M. Alfatni, "Oil palm fruit grading using a hyperspectral device and machine learning algorithm," IOP Conf. Ser. Earth Environ. Sci., vol. 20, p. 012017, Jun. 2014, doi: 10.1088/1755-1315/20/1/012017.

[7] M. K. Shabdin, A. R. M. Shariff, M. N. A. Johari, N. K. Saat, and Z. Abbas, "A study on the oil palm fresh fruit bunch (FFB) ripeness detection by using Hue, Saturation and Intensity (HSI) approach," IOP Conf. Ser. Earth Environ. Sci., vol. 37, p. 012039, Jun. 2016, doi: 10.1088/1755-1315/37/1/012039.

[8] S. Naik and B. Patel, "Machine vision based fruit classification and grading - a review," Int. J. Comput. Appl., vol. 170, no. 9, pp. 22-34, Jul. 2017, doi: 10.5120/ijca2017914937.

[9] O. I. Mba, M.-J. Dumont, and M. Ngadi, "Palm oil: Processing, characterization and utilization in the food industry - A review," Food Biosci., vol. 10, pp. 26-41, Jun. 2015, doi: 10.1016/j.fbio.2015.01.003.

[10] J. Roseleena, J. Nursuriati, J. Ahmed, and C. Y. Low, "Assessment of palm oil fresh fruit bunches using photogrammetric grading system," Int. Food Res. J., vol. 18, no. 3, pp. 999-1005, 2011, available at: Google Scholar.

[11] M. Makky and P. Soni, "Development of an automatic grading machine for oil palm fresh fruits bunches (FFBs) based on machine vision," Comput. Electron. Agric., vol. 93, pp. 129-139, Apr. 2013, doi: 10.1016/j.compag.2013.02.008.

[12] L. C. Lee, C.-Y. Liong, and A. A. Jemain, "Validity of the best practice in splitting data for hold-out validation strategy as performed on the ink strokes in the context of forensic science," Microchem. J., vol. 139, pp. 125-133, Jun. 2018, doi: 10.1016/j.microc.2018.02.009.

[13] P. C. Verpoort, P. MacDonald, and G. J. Conduit, "Materials data validation and imputation with an artificial neural network," Comput. Mater. Sci., vol. 147, pp. 176-185, May 2018, doi: 10.1016/j.commatsci.2018.02.002.

[14] N. Li, X. Zhao, Y. Yang, and X. Zou, “Objects Classification by Learning-Based Visual Saliency Model and Convolutional Neural Network," Comput. Intell. Neurosci., vol. 2016, pp. 1-12, 2016, doi: 10.1155/2016/7942501.

[15] B. Zhou, A. Lapedriza, J. Xiao, A. Torralba, and A. Oliva, "Learning Deep Features for Scene Recognition using Places Database," in Advances in Neural Information Processing Systems 27, Z. Ghahramani, M. Welling, C. Cortes, N. D. Lawrence, and K. Q. Weinberger, Eds. Curran Associates, Inc., 2014, pp. 487-495, available at: Google Scholar.

[16] X. Cheng, J. Lu, J. Feng, B. Yuan, and J. Zhou, “Scene recognition with objectness,” Pattern Recognit., vol. 74, pp. 474-487, Feb. 2018, doi: 10.1016/j.patcog.2017.09.025.

[17] Y. Seo and K. Shin, "Hierarchical convolutional neural networks for fashion image classification," Expert Syst. Appl., vol. 116, pp. 328-339, Feb. 2019, doi: 10.1016/j.eswa.2018.09.022.

[18] J. R. Martinez-Rico, J. Martinez-Romo, and L. Araujo, "Can deep learning techniques improve classification performance of vandalism detection in Wikipedia?," Eng. Appl. Artif. Intell., vol. 78, pp. 248-259, Feb. 2019, doi: 10.1016/j.engappai.2018.11.012. 